\title{
Riesgo y protección de desviación social en adolescentes inmigrantes: Personalidad, familia y aculturación
}

\author{
Jorge Sobral ${ }^{*}$, José Antonio Gómez-Fraguela², Estrella Romero ${ }^{2}$, Ángeles Luengo ${ }^{2}$, Paula Villar $^{2}$ \\ ${ }_{1}$ Departamento de Psicología Social. Universidad de Santiago de Compostela \\ ${ }^{2}$ Departamento de Psicología Clinica y Psicobiología. Universidad de Santiago de Compostela
}

\begin{abstract}
Resumen: En este trabajo se analizan los niveles de conducta antisocial en adolescentes inmigrantes latinoamericanos, residentes en España, integran do tales resultados en un marco explicativo amplio, definido por las interrelaciones de las estrategias de aculturación prevalentes con algunas variables de personalidad y con evaluaciones del funcionamiento familiar. Nuestros resultados alertan acerca de la configuración de ciertas situaciones de especial riesgo ( $\mathrm{y}$, alternativamente, particularmente protectoras) en términos de desviación social en tales sujetos: concretamente, un estilo de separación asociado con bajos niveles de empatía cognitiva y afectiva, pobre autocontrol escasa supervisión parental, baja adaptabilidad y cohesión familiar. Se atiende a las diferencias de sexo/género en todas las variables evaluadas. Se discuten los resultados desde un punto de vista psicosocial, al tiempo que se aboga por prácticas facilitadoras de patrones de integración flexible y bidireccional de inmigrantes, solo posibles cuando la sociedad receptora privilegie unas actitudes de aculturación contrarias al etnocentrismo, al prejuicio, la discriminación y la xenofobia. A lo largo del texto se argumenta acerca de los peligros que pueden acarrear ciertas aproximaciones que parecerían sostener la responsabilidad exclusiva del inmigrante en el éxito adaptativo de su experiencia migratoria.
\end{abstract}

Palabras clave: Aculturación; Personalidad; Familia; Conducta antisocial.

\section{Introducción}

Las migraciones han sido una de las características más relevantes y llamativas de los comportamientos humanos a lo largo de la historia. Se tratan de fenómenos susceptibles de múltiples niveles de análisis, tanto de orden colectivo (político, socioeconómico, etc.) como individual. Sin embargo, y a pesar de su recurrencia a lo largo de los tiempos, parece existir hoy una extendida representación de que las migraciones son más generalizadas, más intensas, y, sobre todo, más problemáticas. Es muy probable que el alto grado de interconexión, de conocimiento mutuo, que han alcanzado las sociedades de nuestros días, estén contribuyendo a esta representación en un doble sentido: en primer lugar, facilitando e incentivando que el fenómeno se produzca (por ejemplo, mostrando a amplias masas empobrecidas las posibilidades de progreso en otras sociedades) $y$, en segundo lugar, haciéndonos más conscientes que nunca de la existencia del fenómeno, cultivando todo un conjunto de actitudes acerca de él; y, finalmente, generando notables sesgos y distorsiones relevantes a los efectos de su evaluación. En otras palabras, la globalización puede haber incrementado los movimientos migratorios tanto como un conjunto de peculiares percepciones sobre ellos.

En cualquier caso, es indudable que cientos de millones de personas están implicadas en estos procesos a lo largo de

* Dirección para correspondencia [Correspondence address]: Jorge Sobral. Departamento de Psicología Social. Universidad de Santiago de Compostela. Campus Sur. 15782-Santiago de Compostela (España). E-mail: jorge.sobral@usc.es
Title: Risk and protection for social deviation in immigrant adolescents: Personality, family, and acculturation.

Abstract: This study analyzes levels of antisocial behavior in Latino immigrant adolescents who are living in Spain. The results are integrated within a wide explanatory framework, defined by the relationships between acculturation strategies and personality variables and family functioning. The results allow us to underline configurations of high risk (and, alternatively, strongly protective) in terms of social deviation: particularly, a separation style linked to low cognitive and affective empathy, poor self-control, low parent supervision, low family adaptability and cohesion. Differences of sex/gender are considered for all the assessed variables. The results are discussed from a psychosocial viewpoint, suggesting the need of promoting patterns of bidirectional flexible integration for immigrants. These patterns would only be feasible when the receiving society favors acculturation attitudes contrary to ethnocentrism, prejudice, discrimination and xenophobia. Throughout the article, the dangers of approaches that hold immigrants exclusively responsible for their adaptive success are pointed out.

Key words: Acculturation; personality; family; antisocial behavior.

todo el mundo; además, muchos indicadores señalan que tales movimientos continuarán creciendo en el futuro (ONU, 2002). Se han constatado ya algunos de los efectos de esta realidad: las minorías etnoculturales se han convertido en una fracción esencial del tejido social de las comunidades de acogida, provocando en ellas cambios de acomodación en muy diversos sentidos y con notables implicaciones. Uno de los resultados de esa situación es el fuerte debate público sobre los derechos de la población inmigrante, con su traslado a la esfera específica de la confrontación política. Algunos han descrito esta situación, entendemos que acertadamente, como encuentro asimétrico entre la búsqueda de legitimidad de los recién llegados y los temores de diverso cuño activados en las poblaciones locales. (Albar et al., 2010). Nacen así los riesgos de conflictividad intercultural y de fragmentación social.

La presencia de población extranjera en España es cada vez más notable, siendo tal vez su incremento el cambio más conspicuo que se ha producido en nuestra sociedad desde hace mucho tiempo. Con ritmos enormemente acelerados, España ha mutado de país emisor de emigración a receptor de inmigración. Por ejemplo, y hablando de jóvenes escolarizados, los datos recogidos en el último Informe de la Juventud en España (INJUVE, 2008), elaborado por el Observatorio de la Juventud, son muy expresivos: la presencia de estudiantes inmigrantes en la educación no universitaria era del $0.54 \%$ en el curso $1992 / 1993$, alcanzando el $8.5 \%$ en el $2006 / 2007$.

Así pues, en España, como en otros muchos países, grandes multitudes de individuos están contribuyendo a crear sociedades cada vez más interculturales, al tiempo que ex- 
perimentan necesariamente el llamado proceso de aculturación. Se trata del concepto más utilizado para referirse a estos procesos friccionales propios de las sociedades multiculturales nacidas al socaire de los procesos migratorios. Ha tenido un gran éxito, sobre todo en tiempos relativamente recientes, aunque fue propuesto ya en 1936, desde una matriz antropológica, por Redfield, Linton y Herskovits para referirse a los cambios resultantes del contacto directo e intenso entre individuos o grupos con distintas culturas. Así, ha sido ampliamente empleado en ciencias sociales para referirse a las mutaciones producidas en colectivos tan diversos como los refugiados políticos, los exiliados de todo tipo, los turistas, los inmigrantes, etc. (Rudmin, 2003).

A pesar de su procedencia desde las llamadas ciencias sociales mayores, o tal vez por eso mismo, el concepto de aculturación ha tenido un notable éxito en el discurso psicológico sobre el fenómeno migratorio. En ese ámbito, se ha convertido en un concepto clave, alrededor del que se han vertebrado modelos de cierto alcance y gran difusión. Tal vez el más conocido y utilizado sea el modelo de Berry (Berry, 1997; Berry, Phinney, Sam y Vedder, 2006). La idea central de esta aproximación sería la existencia de ciertas estrategias con las que los individuos se enfrentan a la experiencia (o, mejor, al amplio conjunto multidimensional de experiencias) que implica el choque cultural implícito en la experiencia migratoria. La estrategia de cada individuo resultaría de un cierto balance de adhesiones entre su cultura de origen vs. su cultura de acogida. Así, y sintetizando, desde esta perspectiva suelen diferenciarse las siguientes estrategias de aculturación: a) Integración (caracterizada por actitudes positivas del inmigrante hacia ambos contextos culturales, el de origen y el de acogida); b) Asimilación (aceptación y predisposición actitudinal positiva hacia el nuevo entorno cultural, al tiempo que rechaza y/o olvida el entorno de origen); c) Separación (disgusto, rechazo ante el entorno de acogida, acompañado de una cierta exaltación idealizada de la cultura de procedencia). En otro trabajo (Sobral, Gómez-Fraguela, Luengo, Romero y Villar, 2010) hemos aludido a esta estrategia como un patrón reactivo de tipo nostálgico, con las implicaciones relativas a procesos internalizantes (ansioso/depresivos, etc.) y externalizantes a que pueda dar lugar; Y, d) Marginación (estrategia en la que predominan actitudes negativas hacia ambos entornos culturales).

A pesar del gran poder seminal de este modelo, y de su probada capacidad para diseminarse a través de la literatura científica, no está exento de críticas. La primera de ellas tendría que ver con un cierto psicologicismo de esta aproximación, en la medida en que haría referencia privilegiada a una serie de estrategias individuales, como si tales estrategias fueran el resultado de una libre elección del inmigrante en un escenario ideal de igualdad entre alternativas. En este enfoque se obviarían las profundas asimetrías de poder a las que estarían sometidos los inmigrantes y se haría pasar por libre albedrío lo que no sería muchas veces más que pura determinación contextual. Además, de manera sutil y probablemente no intencional, se abre paso a la idea de que la integración (como resultado último al que aspirar) depende solo de una de las partes, justo aquella más vulnerable (Rudmin, 2006). En pocas palabras, se ha sugerido que este tipo de modelos ofrecen una sofisticada vía de escape para ignorar las desigualdades de poder, el racismo, la xenofobia y la exclusión social. Es más, se hurtaría el núcleo de un posible análisis diferente, ocultando el rol del grado de control sociopolítico que se estaría negando a las nuevas comunidades en las sociedades de acogida (Hernández-Plaza, García-Ramírez, Camacho y Paloma, 2010).

Tal vez el marco más crítico, y eventualmente productivo, desde el que se haya ofrecido una visión, en cierto sentido alternativa, a los modelos anglosajones dominantes sea el de la denominada Psicología de la Liberación: su propósito, en este ámbito, sería abordar el fenómeno migratorio desde una perspectiva ecológica, centrada en las dinámicas subyacentes de poder que caracterizan las relaciones humanas. (Véase el exhaustivo y documentado trabajo al respecto de Albar et.al., 2010). En ese sentido, nosotros mismos hemos argumentado profusa y vehementemente acerca de los procesos psicosociales y el marco político que fomentan en la Unión Europea la construcción de humanidades de segunda categoría, criminalizando a los inmigrantes que pierden su empleo (Directiva del Retorno de la U E), mercantilizando así sus identidades, y arrojándoles a la marginalidad. Y, si acaso, a la respuesta humillada y violenta (Sobral, Gómez-Fraguela, Luengo y Romero, 2010). Queda abierta así la puerta al complejo mecanismo de enfrentamiento que empieza con la construcción social del enemigo, convertido en exogrupo sobre el que proyectar atribuciones hostiles, sesgos devaluatorios y todo tipo de mecanismos cognitivo-afectivos al servicio de la discriminación (Ramírez, Torrente, Ruiz y Rodriguez, 2007).

Sin embargo, sería injusto olvidar que el propio Berry (Berry, 2005) sitúa en la sociedad de acogida gran parte de la responsabilidad sobre el éxito integrador: de hecho, insiste en que la integración es una opción posible solo si el grupo dominante renuncia a sus pretensiones asimiladoras, muestra aceptación y valoración positiva de la diversidad cultural y presenta bajos niveles de prejuicio étnico. Entre nosotros, algunos trabajos han insistido en la necesaria bidireccionalidad en el enfoque del choque migratorio, insistiendo en el relevante papel de las actitudes de aculturación de los miembros de la sociedad de acogida en el resultado global del proceso, al tiempo que también cabe reconocer en ese ámbito de complejidad la aparición de signos de estrés aculturativo en autóctonos (Ruiz, Torrente y Rodríguez, 2009).

Desde nuestro punto de vista, esta polémica es un ejemplo más del viejo problema de los diferentes niveles de análisis que se pueden adoptar ante fenómenos complejos. Idealmente, no habría porque renunciar a ninguno de ellos. En nuestro caso concreto, cuando utilizamos en este y otros trabajos la terminología de las estrategias de aculturación, lo hacemos asumiendo que no se trata de procesos cognitivos solipsistas, encapsulados, rodeados de vacio social. No se trataría de un acto volitivo, decisional, sino del resultado de un complejo proceso de interacciones entre entornos sociales 
amplios, entornos familiares y grupales, características personales diferenciales, múltiples diferencias puramente casuísticas. En definitiva, no entendemos que, sobre todo en adolescentes, el aparecer caracterizado por el perfil de Integrado o Separado sea un estado mental elegido para percibir el mundo y relacionarse con él, sino más bien un resultado más o menos transitorio y modificable, de influencias de múltiple orden. Asumimos, en este y otros trabajos, el rol morfogenético, estructurante, de los factores sociopolíticos. Pero con igual tesón defendemos la necesidad de indagar en aquellos factores que permiten entender como en la misma sociedad, con las mismas determinaciones sociopolíticas, con familias relativamente homogéneas, unos inmigrantes (adolescentes, en nuestro caso) muestran patrones de interacción con la sociedad de acogida tan diferentes de los de otros. Y, a tales efectos, modelos típicos de la Psicología de la Aculturación como el de Berry, y con las matizaciones efectuadas, siguen siendo de una gran utilidad. Y, precisamente por ello, pretendemos incorporar en el análisis el papel de ciertas diferencias individuales básicas y relevantes y relacionarlas con los estilos de aculturación de los adolescentes de nuestra muestra.

Lo cierto es que estos diferentes estilos de afrontamiento de la experiencia de aculturación han sido puestos en relación con numerosas variables provenientes de muy diversos dominios, tanto del ámbito de la normalidad como de la desviación y la psicopatología. En este último campo han sido especialmente abundantes aquellos trabajos que han vinculado la migración y los trastornos a través del concepto de estrés aculturativo, en la búsqueda de clarificar en lo posible las complejas, y a veces contradictorias, evidencias empíricas disponibles sobre la experiencia migratoria y la prevalencia de depresión, ansiedad, esquizofrenia, etc. (Achotegui, 2002; Bhugra, 2004; Collazos, Qureshi, Antonin y Tomás-Sábado, 2008).

En cualquier caso, y hablando de la asociación inmigrantes-conducta antisocial, y de los abundantes mitos al respecto, es obligado referirnos a que Gómez-Fraguela, Sobral, Luengo, Romero y Villar (2009) ya habían encontrado en un estudio realizado con 2400 adolescentes españoles y latinoamericanos residentes en Galicia y Madrid, menores niveles de conducta antisocial entre inmigrantes que entre nacionales (con la única excepción de las agresiones interpersonales). En un trabajo reciente, Sobral et. al., (2010) se ha informado de la notable concentración de conductas antisociales adolescentes en el grupo caracterizado por el estilo de Separación. En este contexto parece relevante examinar la relación que pudiera existir entre los propios estilos de aculturación y aquellas variables de personalidad que la literatura ha relacionado do modo consistente con conductas antisociales tales como la Búsqueda de Sensaciones, la Impulsividad, la Empatía, etc. (Romero, Luengo y Sobral, 2001; Sobral, Luengo, GómezFraguela, Romero y Villar, 2007; Sobral, Romero, Luengo y Marzoa, 2000). Estas características de personalidad, en interacción con variables contextuales de forma tal que potencian sus efectos (Sobral, Gómez-Fraguela, Romero y Luen- go, 2000) deben jugar un rol relevante, tanto en relación a las conductas antisociales como a la selección de las propias estrategias de aculturación. Parece evidente, entonces, el interés de indagar acerca de la relación que pueda existir entre esas características de personalidad y los propios estilos de aculturación. ¿Existirán marcadores personales de la probabilidad de generar una u otra estrategia de aculturación? Este será uno de los objetivos de este trabajo.

Hablando de adolescentes y conductas desviadas, es evidente el papel que desempeñan una serie de variables del contexto familiar. Existe una amplísima evidencia empírica acerca de los efectos diferenciales de las relaciones afectivas familiares, del nivel y tipo de conflictividad familiar, los niveles de comunicación y confianza intermiembros, el rol protector del apego y, por supuesto, acerca de la influencia de los diferentes estilos disciplinares parentales o pautas de crianza. (Alonso, 2010; Bello, 2011; Sobral, Luengo, Romero y Marzoa, 2000; Torrente y Vazsonyi, 2008; Vazsonyi y Belliston, 2007; Villar, Luengo, Gómez y Romero, 2003). En algún caso, se ha relacionado concretamente el efecto de la inmigración con los procesos familiares y la aparición de trastornos internalizantes y externalizantes (Vazsonyi, Trejos-Castillo y Huang, 2006).

Junto a la familia, el grupo de iguales es el factor contextual que más atención ha suscitado en la investigación sobre factores de riesgo de desviación social en adolescentes. La inserción del chico en grupos con elevada conducta antisocial o consumo de drogas se ha manifestado un consistente e intenso correlato de la propia desviación (Luengo et al., 1994). Ahora bien, permanece la discusión acerca de la dirección de la causalidad: tal vez el grupo conforme al individuo en su peculiar entorno normativo-conductual, asimilándole y homogeneizándole, o, al contrario, es posible que ciertas predisposiciones personales del adolescente impliquen una búsqueda activa por su parte, es decir, una selección de aquel grupo que resulte consistente con sus particularidades idiosincráticas. Por ejemplo, en un estudio longitudinal con adolescentes españoles, Romero, Luengo y Gómez-Fraguela (2000) encontraron evidencia empírica congruente con la simultaneidad de ambos procesos: los adolescentes con actitudes y conductas problemáticas tendieron a seleccionar amigos similares, al tiempo que los procesos intragrupales de influencia normativa y modelado reforzarían la implicación en tales conductas.

Antes de explicitar los objetivos concretos de este trabajo, conviene resaltar que: a) por una parte, la inmensa mayor parte de los estudios que han abordado diferentes asuntos relativos a inmigrantes y sus experiencias ante el choque migratorio, se han hecho con poblaciones en las que la necesidad de aprender un nuevo idioma juega un rol decisivo en la aculturación. Tanto es así que, en numerosos estudios, se ha equiparado en gran medida el aprendizaje del nuevo idioma con el criterio básico para determinar el grado de integración alcanzado (Marsiglia, Kulis, Wagstaff, Elek y Dran, 2005; Zarza y Sobrino, 2007). Nuestro trabajo es uno de los pocos en los que la lengua de la sociedad de origen y la de acogida 
coinciden. $\mathrm{Y}, \mathrm{b}$ ) es interesante recordar aquí la escasez de estudios insertos en este marco conceptual realizados con adolescentes. Se ha dedicado mucho esfuerzo a analizar los efectos del choque migratorio-cultural en adultos, y mucho menos en sus hijos. Es razonable pensar que la experiencia aculturativa tenga matices peculiares en una etapa evolutiva tan peculiar como la adolescencia (es probable que la tensión generada por la experiencia migratoria se añada a las tensiones típicas de esta edad). En definitiva, nuestra aportación empírica aporta la doble peculiaridad de una perspectiva centrada en adolescentes sin choque idiomático.

Así pues, el objetivo general de este trabajo sería analizar las diferencias existentes entre adolescentes inmigrantes con distintos estilos de aculturación en una serie de variables psicosociales. Más específicamente, a) queremos comprobar si alguna/s variable de personalidad se asocia significativamente con la adscripción a uno u otro estilo de aculturación. Parece prometedor averiguar si existen perfiles personales que faciliten estilos de afrontamiento más saludables de la experiencia aculturativa; b) queremos conocer cuál es la asociación que se establece entre los diferentes estilos de aculturación y ciertas variables de funcionamiento intrafamiliar; c) aunque asumimos que la literatura disponible avala sobradamente la utilidad de las variables anteriores como indicadores indirectos del riesgo de transgresión adolescente, analizaremos la relación que las diferentes estrategias de aculturación establezcan con medidas directas de algunas conductas antisociales, incluido el consumo de drogas; d) esperamos que los resultados parciales nos permitan su vertebración en un marco general, con sus aspectos personales y contextuales, acerca de los factores de riesgo y protección en adolescentes inmigrantes; $\mathrm{Y}$ e) nos interesa señalar que, en atención a las notables y bien establecidas diferencias sexuales en el ámbito de las conductas antisociales adolescentes (y de la delincuencia adulta), en todos los análisis se constatarán tales diferencias.

\section{Método}

\section{Participantes}

Los participantes en el estudio fueron reclutados en distintos centros públicos de Educación Secundaria Obligatoria (ESO) de las Comunidades Autónomas de Galicia y Madrid. En Galicia se seleccionaron los 21 centros con mayor número de inmigrantes de entre todos los centros de la Comunidad (se utilizó como fuente los datos estadísticos proporcionados por la Consellería de Educación de la Xunta de Galicia). En la Comunidad de Madrid se recogieron datos en nueve centros de tres localidades con notable presencia de inmigrantes latinoamericanos. Para este estudio sólo se han considerado aquellos casos en los que tanto los adolescentes como sus padres y madres habían nacido en algún país latinoamericano. La muestra final estuvo compuesta por 750 adolescentes procedentes de 15 países. Los colectivos más numerosos procedían de Ecuador (35\% de la muestra) y Co- lombia $(20.5 \%)$, siendo minoritarios los procedentes de la República Dominicana (7.5\%), Argentina (7.1\%), Perú $(6.7 \%)$, Bolivia $(5.9 \%)$ y Uruguay $(5.2 \%)$. De los 750 casos seleccionados, 477 se reclutaron en Madrid y 273 en Galicia. Finalmente, el $53.4 \%$ fueron varones y el $46.6 \%$ mujeres, siendo su edad media de 14.3 años (con un rango comprendido entre los 11 y los 17 años).

\section{Instrumentos}

Para categorizar a los adolescentes inmigrantes según el estilo de aculturación utilizado analizamos distintas variables. Para evaluar la atracción por la cultura de origen o de acogida empleamos una versión del Cuestionario de Implicación Cultural (BIQ), elaborado por Szapocznik, Kurtines y Fernández (1980). En este autoinforme se pide a los adolescentes que valoren aquellos aspectos propios de la cultura de origen y de acogida (costumbres, músicas, actividades de ocio, medios de comunicación, tradiciones familiares, etc.). Para este estudio eliminamos los ítems referidos al idioma, utilizando una versión de 15 ítems que los sujetos debían contestar en una escala de cinco alternativas (nada, poco, algo, bastante y mucho). La consistencia interna para las dos escalas fue elevada $(\alpha=.91$ para la vinculación con el país de origen $\mathrm{y} \alpha=.92$ para la vinculación con el país de acogida). Otra variable analizada fue el grado en que los adolescentes se identificaban como españoles o como personas de su país de origen. Para esto preguntamos cuánto sentían que tenían en común con un español y cuánto con alguien de su país de origen. La escala de respuestas incluía cinco alternativas (nada, casi nada, poco, bastante y mucho). Una tercera variable considerada fue el tipo de personas con las que se relacionaban. Les preguntamos sobre la cantidad de amigos que habían nacido en España (ninguno, casi ninguno, algunos, la mitad y todos o casi todos), por el tipo de amigos con los que se relacionaban más frecuentemente en el colegio y fuera de él y por el tipo de personas que solían visitar su hogar. Las alternativas de respuestas en estos casos también se presentaron en una escala de cinco puntos (exclusivamente de otro país, la mayoría de otro país, mitad y mitad, la mayoría españoles, exclusivamente españoles). Con estas cuatro preguntas creamos un índice global sobre la cantidad de amigos españoles. La consistencia interna de esta puntuación fue elevada $(\alpha=.78)$.

Para evaluar la Impulsividad se recurrió a la escala de Impulsividad del cuestionario I6 elaborado originalmente por Eysenck, Easting y Pearson (1984) y adaptada en España por Silva, Martorell y Clemente (1987). La consistencia interna obtenida en este estudio fue de $\alpha=.80$. Para evaluar la Búsqueda de Sensaciones se utilizo la escala de 10 ítems incluida en el cuestionario del ZKPQ-III (Romero, Luengo, Gomez-Fraguela, y Sobral, 2002; Zuckerman, Kuhlman, Joireman, Teta y Kraft, 1993), presentando una consistencia interna de $\alpha=.73)$. En los dos casos las alternativas de respuesta utilizadas fueron dicotómicas (si/no). Para evaluar la dimensión de Empatía se ha utilizado la Basic Empathy Scale (Jolliffe y Farrington, 2006). Este instrumento está com- 
puesto por 20 ítems que se deben contestar en una escala con cinco alternativas de respuesta en las que deben manifestaren qué grado las afirmaciones se ajustan a uno mismo (desde muy en desacuerdo a muy de acuerdo). Además de una puntuación global, con este instrumento se puede obtener una puntuación en Empatía Cognitiva y otra en Empatía Afectiva. La consistencia interna para las dos dimensiones fue de $\alpha$ $=74 \mathrm{y} \alpha=.65$ respectivamente.

Para evaluar las variables familiares se utilizó una versión reducida del Cuestionario de Conducta Conflictiva (CBQ; Conflict Behavior Questionnaire, Prinz, Foster, Kent y O'Leary, 1979) para el nivel de conflictividad. Este instrumento consta de 20 ítems y evalúa la información aportada por los adolescentes acerca de las interacciones positivas y negativas que mantienen con sus padres. Los ítems se contestan en un formato de tres alternativas (nunca, algunas veces $\mathrm{y}$ siempre) y la consistencia interna obtenida con los inmigrantes fue de .89. Para evaluar la cohesión familiar y la adaptabilidad familiar percibida por los adolescentes se utilizó una versión reducida del Family Adaptability and Cohesion Evaluation Scale (FACES II)(Olson, Bell y Portner, 1982). La versión empleada consta de 22 ítems (11 en cada dimensión) que se deben contestar en qué medida cada pregunta es aplicable a su familia. Para ello se utiliza una escala de cinco puntos que va desde casi nunca (0) a casi siempre (4). La consistencia interna de la escala de cohesión fue de $\alpha=.86$ y de $\alpha$ $=.75$ para la escala de adaptabilidad. Para evaluar los niveles de supervisión familiar se empleó una escala de elaboración propia de 6 ítems (e.g. Tus padres saben qué haces en tu tiempo libre) que reflejan la percepción de los adolescentes relativa al grado en que sus padres ejercen vigilancia sobre ellos, establecen normas y guían su conducta (Villar, Luengo, GómezFraguela y Romero, 2006). En este caso también se presentaban 5 alternativas de respuesta (desde casi nunca a casi siempre). La consistencia interna fue de $\alpha=.66$.

Para evaluar la conducta antisocial se utilizó una versión reducida del Cuestionario de Conducta Antisocial (CCA; Luengo, Otero, Romero, Gómez-Fraguela y Tavares-Filho, 1999). Consta de diversas preguntas acerca de la frecuencia con la que, en los últimos 12 meses, ha realizado el adolescente diversas conductas transgresoras: a) agresiones interpersonales (p.ej., promover peleas con otros); b) vandalismo (p.ej., destrucción intencionada de mobiliario urbano); c) conductas antinormativas (p.ej., pasar la noche fuera de casa sin permiso); d) hurtos (p.ej., en tiendas), y e) problemas relacionadas con las drogas (conductas ilegales dirigidas a conseguir drogas y/o traficar con ella). Las alternativas de respuesta incluyen 4 opciones (nunca, pocas veces - de 1 a 5 veces, bastantes veces - de 5 a 10, o con frecuencia- 10 veces o más) y la consistencia interna en las distintas escalas fue de .80 para la escala de agresión, .77 para vandalismo, .73 en las conductas antinormativas, .50 para robo y problemas con las drogas.

\section{Procedimiento}

La investigación fue presentada a los equipos directivos de los centros educativos seleccionados. Se les explicaron los aspectos fundamentales de la misma, sus objetivos, al tiempo que se solicitaba su colaboración. Todos los centros se mostraron dispuestos a colaborar. Después de solicitar el consentimiento paterno para la participación de los adolescentes en la investigación, se invitó a participar a todos los estudiantes escolarizados en el centro (nativos e inmigrantes).. La aplicación de las pruebas se efectuó de manera colectiva en las aulas, durante el horario escolar y en una sesión de 60 minutos. Las pruebas fueron aplicadas por miembros del grupo de investigación que en todo momento garantizaron el anonimato de las respuestas y la voluntariedad en la participación. Los docentes del centro estuvieron ausentes en todo momento.

\section{Análisis de datos}

Para agrupar a los inmigrantes en función de la estrategia de aculturación adoptada se realizó un análisis de conglomerados (método de Kmedias). Para realizar el análisis se utilizaron las puntuaciones típicas obtenidas en el BIQ, el grado en que se identificaban como españoles o como personas de su país de origen y el tipo de amigos con los que se relacionaban (Hair, Anderson, Tatham y Black, 1999).

Para analizar si existían diferencias entre los grupos de inmigrantes en las distintas variables analizadas se realizaron de forma separada para varones y mujeres Análisis de $\mathrm{Va}$ rianza Multivariados (MANOVAS) utilizando el grupo de aculturación como variable independiente. Para analizar entre que grupos se producían las diferencias significativas se utilizó la prueba de Sheffé (nivel $\alpha$ de .05). Todos los análisis fueron realizados con el paquete estadístico PASW Statistics 18.

\section{Resultados}

En la Figura 1 se representan las puntuaciones medias obtenidas en esas variables para los cuatro conglomerados solicitados. El conglomerado más numeroso es el número dos, en el que se agrupan 303 casos. Este grupo está formado por aquellos inmigrantes que adoptan una estrategia de integración en el proceso de aculturación (alta atracción por la cultura de origen y de acogida, tienen mucho en común con los españoles y con las personas de su nacionalidad y se relacionan con bastantes amigos del país de acogida). El conglomerado cuarto le sigue en tamaño y en él se incluyen 207 casos. Las características de este conglomerado permiten identificarlos con la estrategia de separación (puntuaciones elevadas en la escala de atracción por su cultura de origen, alta identificación con las personas procedentes de su país, bajas puntuaciones en la atracción por la cultura de acogida, escasa identificación con los españoles y muy escasa relación con amigos españoles). En el conglomerado número tres se agrupan 
143 adolescentes. Su perfil permite identificar a sus miembros con aquellos que optan por una estrategia de asimilación (aunque se sienten poco atraídos por la cultura de acogida, sienten que tienen mucho en común con los españoles y poco con las personas de su país de origen y son los que más se relacionan con otros amigos españoles). Por último, el con- glomerado uno es el menos numeroso (97 inmigrantes). La escasa identificación con los españoles y con las personas de su país, las escasas relaciones con compañeros españoles y la atracción intermedia por la cultura de acogida y de su país de origen, indican que los casos agrupados en este conglomerado se acercarían al perfil de marginación.

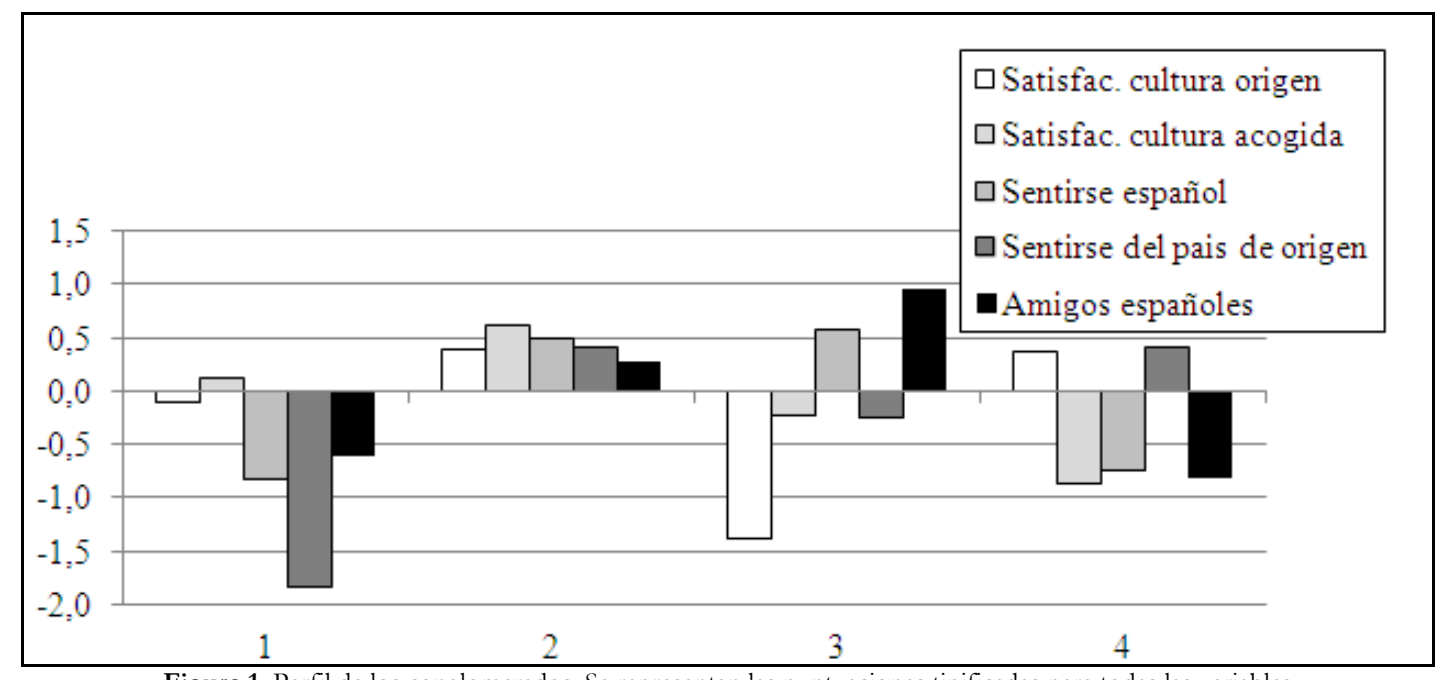

Figura 1. Perfil de los conglomerados. Se representan las puntuaciones tipificadas para todas las variables

En la Tabla 1 se presentan las diferencias que se observaron entre los grupos de chicos y chicas con distintos estilos de aculturación en las variables de personalidad.

En el caso de los varones se obtuvieron diferencias significativas entre los grupos de aculturación para las dos escalas de empatía. En el primer caso, la empatía cognitiva, las diferencias se concentran entre el grupo de marginación, que presenta las puntuaciones más bajas, y el grupo de integración, con los valores más elevados. En el caso de la empatía afectiva, las diferencias se producen entre el grupo de mar- ginados y los otros tres. Para los grupos de mujeres las diferencias en personalidad se producen en las escalas de empatía cognitiva y en la escala de impulsividad. En el primer caso también son las adolescentes incluidas en el grupo de marginación las que presentan valores más bajos en empatía (diferenciándose significativamente de los otros tres grupos). En cuanto a la impulsividad, son las que optan por las estrategias de marginación y separación las que presentan puntuaciones medias más elevadas que las del grupo de integración.

Tabla 1. Diferencias en función del estilo de aculturación en las variables de personalidad para varones y mujeres.

\begin{tabular}{|c|c|c|c|c|c|c|}
\hline & $\begin{array}{c}\text { Marginación } \\
M(D T)\end{array}$ & $\begin{array}{c}\text { Integración } \\
M(D T)\end{array}$ & $\begin{array}{c}\text { Asimilación } \\
M(D T)\end{array}$ & $\begin{array}{c}\text { Separación } \\
M(D T)\end{array}$ & $F$ & $p$ \\
\hline Varones & $(n=58)$ & $(n=150)$ & $(n=94)$ & $(n=100)$ & & \\
\hline Empatía cognitiva & $17.77(5.6)$ & $24.39(6.8)$ & $22.44(5.0)$ & $21.84(6.9)$ & 4.83 & .003 \\
\hline Empatía afectiva & $16.90(4.8)$ & $23.53(5.9)$ & $22.34(4.8)$ & $23.69(6.1)$ & 4.87 & .003 \\
\hline Búsqueda sensaciones & $5.53(2.3)$ & $5.18(2.4)$ & $4.76(2.4)$ & $5.85(2.0)$ & 1.74 & .162 \\
\hline Impulsividad & $10.59(3.5)$ & $10.86(4.9)$ & $9.73(4.9)$ & $11.01(4.2)$ & 0.95 & .420 \\
\hline Mujeres & $(n=39)$ & $(n=153)$ & $(n=49)$ & $(n=107)$ & & \\
\hline Empatía cognitiva & $18.64(10.1)$ & $26.65(6.0)$ & $25.80(6.2)$ & $26.08(5.0)$ & 6.12 & .001 \\
\hline Empatía afectiva & $26.83(4.3)$ & $29.04(6.8)$ & $27.18(5.6)$ & $28.15(6.0)$ & 1.15 & .333 \\
\hline Búsqueda sensaciones & $5.71(2.0)$ & $4.73(2.5)$ & $4.60(2.7)$ & $5.41(2.2)$ & 1.09 & .358 \\
\hline Impulsividad & $13.20(4.1)$ & $9.90(4.8)$ & $10.30(6.0)$ & $12.41(4.9)$ & 3.20 & .025 \\
\hline
\end{tabular}

Los resultados de comparar los grupos en las distintas variables psicosociales se presentan en la tabla 2. Las diferencias encontradas entre los grupos de aculturación en varones se centran en las variables de cohesión y supervisión familiar. Concretamente es el grupo de integrados el que presenta puntuaciones medias superiores tanto en la escala de cohesión como en la de supervisión familiar. El grupo con el nivel de cohesión familiar más bajo sería el de asimilación, siendo el de separación el que presenta menores niveles de supervisión familiar. En la muestra de mujeres las diferencias significativas se producen en las escalas de adaptabilidad, cohesión y supervisión familiar. En los dos primeros 
casos es el grupo de muchachas con una estrategia de aculturación integrada el que presenta puntuaciones más elevadas. En el caso de supervisión la media superior se halla en el grupo de marginación. En las tres variables los valores inferiores se encuentran en el grupo de separación.

Tabla 2: Diferencias en función del estilo de aculturación en las variables familiares y escolares para varones y mujeres

\begin{tabular}{|c|c|c|c|c|c|c|}
\hline & $\begin{array}{c}\text { Marginación } \\
M(D T)\end{array}$ & $\begin{array}{c}\text { Integración } \\
M(D T)\end{array}$ & $\begin{array}{c}\text { Asimilación } \\
M(D T)\end{array}$ & $\begin{array}{c}\text { Separación } \\
M(D T)\end{array}$ & $F$ & $p$ \\
\hline Varones & $(n=58)$ & $(n=150)$ & $(n=94)$ & $(n=100)$ & & \\
\hline Conflicto familiar (CBQ) & $19.36(12.7)$ & $21.04(11.4)$ & $21.05(11.3)$ & $22.07(11.8)$ & 0.27 & .844 \\
\hline Adaptabilidad (FACES) & $31.85(7.4)$ & $32.24(7.0)$ & $30.50(5.9)$ & $30.78(6.9)$ & 0.99 & .399 \\
\hline Cohesión (FACES) & $29.03(8.5)$ & $31.26(6.7)$ & $28.06(7.5)$ & $28.41(8.2)$ & 3.91 & .009 \\
\hline Superv. Familiar & $14.42(5.2)$ & $15.72(3.7)$ & $14.82(4.3)$ & $14.35(4.3)$ & 2.70 & .046 \\
\hline Mujeres & $(n=39)$ & $(n=153)$ & $(n=49)$ & $(n=107)$ & & \\
\hline Conflicto familiar (CBQ) & $19.12(12.7)$ & $20.39(11.9)$ & $22.35(12.8)$ & $24.27(12.5)$ & 1.95 & .121 \\
\hline Adaptabilidad (FACES) & $31.13(7.0)$ & $33.48(7.2)$ & $30.84(8.0)$ & $30.03(8.1)$ & 3.38 & .019 \\
\hline Cohesión (FACES) & $29.68(8.2)$ & $30.78(8.5)$ & $27.76(8.4)$ & $27.21(8.5)$ & 2.69 & .047 \\
\hline Superv. Familiar & $16.87(3.2)$ & $16.54(3.7)$ & $16.37(3.3)$ & $15.34(3.9)$ & 2.63 & .050 \\
\hline
\end{tabular}

Para finalizar, comparamos los inmigrantes en la frecuencia con que se implicaban en distintos tipos de conductas antisociales (Tabla 3). En los inmigrantes varones se obtienen diferencias significativas en las conductas de agresión y en los problemas con el consumo de drogas. En ambos casos es el grupo de separación el que presenta una mayor implicación con ese tipo de conductas. Para las mujeres, las diferencias encontradas solo alcanzan niveles de significación estadística en el caso de las conductas contra normas, siendo también en ellas el grupo con estrategias de aculturación centradas en la separación el que presenta una mayor implicación con este tipo de conductas.

Tabla 3: Diferencias en función del estilo de aculturación en la conducta antisocial para varones y mujeres

\begin{tabular}{|c|c|c|c|c|c|c|}
\hline & $\begin{array}{c}\text { Marginación } \\
M(D T)\end{array}$ & $\begin{array}{c}\text { Integración } \\
M(D T)\end{array}$ & $\begin{array}{c}\text { Asimilación } \\
M(D T)\end{array}$ & $\begin{array}{c}\text { Separación } \\
M(D T)\end{array}$ & $F$ & $p$ \\
\hline Varones & $(n=58)$ & $(n=150)$ & $(n=94)$ & $(n=100)$ & & \\
\hline Agresión & $1.19(1.2)$ & $1.40(2.3)$ & $0.38(0.7)$ & $2.67(2.8)$ & 5.24 & .002 \\
\hline Vandalismo & $0.83(1.0)$ & $1.10(2.4)$ & $0.50(1.1)$ & $1.63(1.9)$ & 1.41 & .241 \\
\hline Cond. Contra normas & $1.40(2.2)$ & $1.97(2.9)$ & $0.99(1.7)$ & $2.75(2.1)$ & 1.45 & .229 \\
\hline Robo & $1.67(0.4)$ & $0.44(1.2)$ & $0.27(0.7)$ & $0.65(1.2)$ & 1.39 & .247 \\
\hline Problemas drogas & $0.17(0.4)$ & $0.83(1.1)$ & $0.52(1.1)$ & $1.65(1.7)$ & 2.83 & .040 \\
\hline Mujeres & $(n=39)$ & $(n=153)$ & $(n=49)$ & $(n=107)$ & & \\
\hline Agresión & $0.25(0.5)$ & $0.35(0.7)$ & $0.68(1.6)$ & $1.24(2.2)$ & 2.28 & .082 \\
\hline Vandalismo & $0.06(0.4)$ & $0.09(0.3)$ & $0.17(0.5)$ & $0.43(1.0)$ & 2.30 & .079 \\
\hline Cond. Contra normas & $0.80(1.8)$ & $0.93(1.4)$ & $1.78(2.4)$ & $2.437(2.1)$ & 3.18 & .026 \\
\hline Robo & $0.11(0.4)$ & $0.32(0.7)$ & $0.32(0.7)$ & $0.14(0.4)$ & 0.57 & .636 \\
\hline Problemas drogas & $0.80(1.8)$ & $0.79(1.5)$ & $0.79(1.7)$ & $1.32(1.2)$ & 0.42 & .737 \\
\hline
\end{tabular}

\section{Discusión y conclusiones}

Aunque pretendemos hacer una discusión integrada, y necesariamente selectiva, de los diferentes resultados de este trabajo, uno de ellos llama poderosamente la atención, por su magnitud cuantitativa y su relevancia cualitativa: nos referimos a la Empatía (Tabla 1). Tanto en su versión cognitiva como afectiva (capacidad para percibir y entender, para conocer, los procesos mentales y emocionales ajenos, así como capacidad para identificarse vicariamente, en algún grado, con las emociones de otros), nuestros resultados vinculan a la capacidad empática con la estrategia más saludable de aculturación (la Integración), al tiempo que nos alertan de nuevo de las llamativas y bien conocidas diferencias de sexo/género en esta variable (superiores puntuaciones de las chicas tanto en empatía cognitiva como en empatía afectiva, resultado probablemente de complejas interacciones entre disposiciones bioconstitucionales y mecanismos de socializa- ción diferencial en el manejo interpersonal de las emociones). Al mismo tiempo, son bien conocidas las funciones inhibidoras de la empatía respecto a las conductas antisociales y su papel facilitador de las prosociales. Además, son muchas las ocasiones en que se ha informado de los fuertes vínculos que establecen las capacidades empáticas con otras características; concretamente, y trabajando con adolescentes, se ha informado, por ejemplo, de sus positivas relaciones con cordialidad, asertividad, autoestima, conciencia y apertura a la experiencia, así como con ciertas medidas de inteligencia general, más elevado estatus socioeconómico y, en el ámbito familiar, una mejor supervisión parental (Jolliffe y Farrington, 2006). Nuestros resultados se pueden insertar en ese mismo patrón de hallazgos, si bien informan adicionalmente del rol esencial de la Empatía en la consecución de mejores resultados en el afrontamiento de la experiencia migratoria. De hecho, si hay algo esencial en el concepto de empatía es su naturaleza relacional. Y la experiencia aculturativa implica per 
se una dinámica relacional. No debería sorprendernos, pues, que un marcador personal significativo de aquellos inmigrantes que han desarrollado un patrón de Integración sean sus capacidades para entender y sintonizar con los otros: percibir las demandas de los autóctonos, ajustarse a ellas sin renegar de sus marcos de procedencia, negociar una posición de equilibrio entre esos universos socioculturales. Es muy probable, además, y habida cuenta de la ya señalada bidireccionalidad de las dinámicas de aculturación, que tal disposición empática genere una cierta reciprocidad en autóctonos: comprenderán mejor al otro al sentirse comprendidos por él. Solo así son posibles las posteriores interacciones basadas en la confianza mutua con sus iguales autóctonos e inmigrantes. Es evidente que si analizamos estas dinámicas desde un punto de vista de la conflictividad intergrupal (el más ad hoc para los movimientos migratorios), los patrones empáticos de interacción reducirían drásticamente fenómenos tan perniciosos y conocidos como el favoritismo endogrupal y la devaluación del exogrupo. De hecho, el análisis de las dinámicas de percepción y estereotipos, subyacentes a los problemas de discriminación y etnocentrismo, forman parte esencial de aquellos programas para adolescentes elaborados con el propósito de estimular el desarrollo socio-emocional general, y el de la empatía en particular (Garaigordobil, 2000; Garaigordobil y García de Galdeano, 2006). Desde un punto de vista negativo (Tabla 1), es especialmente llamativo, aunque con plena coherencia teórica, por comparación con el de los Integrados, el bajo nivel empático de los adolescentes varones Marginados (rechazo al entorno de acogida y del entorno de procedencia).

Otro aspecto relevante en este contexto, con el que se ha relacionado la Empatía es el del grado en que somos hábiles para regular adecuadamente nuestras conductas apetitivas, de aproximación, mediante mecanismos de activación/inhibición, para generar buenos niveles de autocontrol. Pues bien, en el ámbito de las variables de personalidad que hemos analizado, debemos señalar el resultado relativo al papel de la Impulsividad en las mujeres, como marcador personal en el grupo de separados y marginados. La relación de la Impulsividad/Autocontrol con conductas antisociales se ha documentado en muy diversos contextos (Por ejemplo, con adolescentes españoles, Sobral et. al., 2000). Así pues, es razonable pensar que fomentando las capacidades empáticas estaremos incrementando las probabilidades de integración, mejorando el autocontrol y reduciendo el riesgo de conductas antisociales.

Los resultados relativos a la empatía habría que analizarlos en relación con aquellos acerca de las percepciones de los adolescentes de nuestra muestra sobre su entorno familiar (Tabla 2). Es obvio que individuos más empáticos facilitan la producción de mejores relaciones interpersonales en el seno familiar, al tiempo que estas refuerzan las disposiciones empáticas.

Parece claro, analizados en conjunto, que los adolescentes categorizados como separados informan de un conjunto de percepciones, en términos relativos frente a los integrados, sensiblemente más problemáticas. Sus percepciones reflejan un panorama canónicamente asociado con conductas problemáticas de diverso orden: niveles bajos de Adaptabilidad, con todo lo que ello implica de dificultades para la acomodación a las circunstancias cambiantes, de escasa flexibilidad cognitiva, de dificultades para la adaptación y/o generación normas adecuadas a los contextos dinámicos, así como de escasa disposición de estrategias actitudinales (cognitivoafectivas y conductuales) que permitan afrontar eventos frustrantes. Ello se acompaña de percepciones acerca de unos entornos familiares caracterizadas por niveles notablemente bajos de Cohesión, lo cual nos alerta de problemas en cuanto a las necesidades del adolescente de sentir la cercanía de los demás miembros de la familia, de notables problemas a la hora de disponer de canales de comunicación fluidos en relación a conflictos y emociones; además, la baja cohesión suele correlacionarse con mayores dosis de conflictividad y una percepción de escasa disponibilidad de apoyo intrafamiliar. Todas estas características han sido inequívocamente relacionadas con numerosos problemas internalizantes y externalizantes en adolescentes (véase la revisión al respecto de Bello, 2011).

En el mismo contexto, es llamativa la fuerte asociación entre la estrategia de aculturación de Separación en varones y su percepción relativa a la baja Supervisión parental. Así, hay una fuerte diferencia entre los niveles de supervisión parental informados por los Integrados y los Separados. Son bien conocidas las relaciones entre la deficiente supervisión parental y multitud de conductas problema en adolescentes, sobre todo cuando se asocia con débiles vínculos afectivos en la unidad familiar. La supervisión, entendida como control positivo, racional, inserto en un clima familiar autorizativo y dialogante, suele ser un correlato notable de ajuste/desajuste psicosocial en adolescentes. Además, los niveles de supervisión percibidos son notablemente más altos en chicas dentro del grupo de separados, lo cual es coherente con las tendencias de socialización clásicas, tendentes a reforzar el control, en múltiples manifestaciones, sobre las hijas adolescentes.

Por lo que se refiere a las conductas antisociales evaluadas, es interesante señalar que de nuevo nos encontramos con su mayor prevalencia en varones (específicamente en aquellas conductas que implican un mayor nivel de proactividad, a saber agresiones y vandalismo). Pero sobre todo y para, finalmente, completar nuestro planteamiento, merecen un comentario adicional las relaciones halladas entre estrategias de aculturación y las conductas antisociales evaluadas (Tabla 3). En primer lugar, es muy notable la menor concentración de agresiones, conductas antinormativas y de problemas con las drogas, en el grupo de asimilados varones. Probablemente, ello deba ser comprendido en relación con la fuerte presión hacia la adaptación normativa ejercida en el contexto intrafamiliar. Un apoyo empírico a esta interpretación procede de la notable supervisión parental informada por los chicos categorizados en este grupo.

Como se puede observar, entre las relaciones significativas destaca la fuerte asociación entre la pertenencia al grupo 
de separados (tanto varones como mujeres) y la tendencia a participar en agresiones interpersonales, a conductas contra normas y a tener problemas con las drogas. Así pues, resulta evidente que una lectura integral de los datos de este trabajo sugiere un claro perfil psicosocial en el que la Separación (disgusto hacia el entorno español, sentimientos de perdi$\mathrm{da} /$ nostalgia hacia el entorno de procedencia) es especialmente desadaptativo: se concentran ahí, por comparación con el grupo de integrados, menores niveles de Empatía cognitiva, con todas las implicaciones antes sugeridas, un deficitario manejo en el autocontrol implícito en los niveles de Impulsividad (sobre todos en mujeres), los elementos familiares disfuncionales (problemas con la Supervisión, la Adaptabilidad y la Cohesión), los más altos niveles de Conductas Antisociales varias.

Parece evidente, pues, que un trabajo prioritario en este campo sería el desarrollo de intervenciones dirigidas a posibilitar una apertura del adolescente separado hacia el entorno de acogida sin merma alguna de su estima y aprecio por su entorno de procedencia. Obviamente, gran parte de la responsabilidad del éxito/fracaso de este proceso, como ya se ha dicho, radica en las propias actitudes de la sociedad de acogida. Revertir los estilos de separación en lo que podríamos llamar integración flexible: apertura a lo nuevo y sus experiencias, actitud empática de comprensión hacia sus demandas, combinada con la asertividad y el autoconcepto adecuados para mantener el aprecio por las propias tradiciones y costumbres; flexibilidad cognitiva para efectuar la siempre compleja y delicada negociación entre la adhesión al mundo del que se procede y sus lealtades y la inmersión en aquel al que se

\section{Referencias}

Achotegui, J. (2002). La depresión en los inmigrantes: una perspectiva transcultural. Barcelona: Ediciones Mayo.

Albar, M. J., García, M., El Karkri, M., Luque, V., Paloma, V., Camacho, C., El Jebari, T., Hernández, S., Paloma, O. y Sevillano, J.M. (2010). Contribuciones de la Psicología de la Liberación a la integración de la población inmigrante. Intervención Psicosocial, 19, 3, 223-234.

Alonso, C. (2010). Personalidad parental, estilos educativos y problemas de conducta infanto-juveniles. Tesis de Licenciatura, Universidad de Santiago de Compostela.

Bello, I. (2011). Variables familiares y problemas de conducta: estudio de la Escala de Procesos Familiares en Adolescentes. Tesis de Licenciatura, Universidad de Santiago de Compostela.

Berry, J. W. (1997). Immigration, acculturation and adaptation. Applied Psychology: An International Review, 46, 5-68.

Berry, J. W. (2005). Acculturation: living succesfully in two cultures. International Journal of Intercultural Relations, 29, 697-712.

Berry, J. W., Phinney, J. S., Sam, D. L., \& Vedder, P. (2006). Immigrant youth in cultural transition: Acculturation, identity and adaptation across national contexts. Mahwah, NJ: Lawrence Erlbaum Associates.

Bhugra, D. (2004). Migration and mental health. Acta Psychiatrica Scandinavica, 108 (Supl. 418), 67-72.

Collazos, F., Qureshi, A., Antonin, M. y Tomás-Sábado, J. (2008). Estrés aculturativo y salud mental en la población inmigrante. Papeles del Psicólogo, 29(3), 307-315.

Eysenck, S. B., Easting, G., \& Pearson, P. R. (1984). Age norms for impulsiveness, venturesomeness and empathy in children. Personality and Individual Differences, 5(3), 315-321. llega. En la medida en que seamos capaces de generar y/o facilitar ese círculo virtuoso (sensibilidad interpersonal en el manejo de las emociones, autocontrol, buen funcionamiento familiar: flexibilidad, afecto, comunicación y supervisión o control positivo; contacto grupal con otros similares bien adaptados, y los múltiples feedbacks positivos entre todas esas variables) estaremos fomentando en adolescentes inmigrantes un saludable ajuste social y psicológico.

Por supuesto, este patrón de resultados tendría que ser confirmado con estudios que dispongan de otras muestras, con sujetos de otras procedencias geográficas y culturales, seleccionadas de entornos de mayor exclusión social, con registros delictivos oficiales, eventualmente más graves y no solo autoinformados. Además, es necesario progresar en la dirección de identificar variables no solo reveladoras del desajuste, sino también en el sentido de elaborar perfiles de éxito y resiliencia ante la experiencia migratoria. Y nunca se puede olvidar la imperiosa necesidad de generar discursos sociales integradores, constructores de diques contra el etnocentrismo, los prejuicios, el racismo y la xenofobia. Este trabajo pretende ser una contribución a ese proyecto, definido en último término por lo que hemos denominado paradigma de la utopía mínima (Sobral, Gómez-Fraguela, Luengo y Romero, 2010): ante la amenaza de licuación de ciertos valores, la radical reivindicación de la igualdad de los seres humanos.

Nota.- La investigación descrita en este trabajo ha sido subvencionada por el Ministerio de Educación y Ciencia (proyecto SEJ200508949).

Garaigordobil, M. (2000). Intervención psicológica con adolescentes. Un programa para el desarrollo de la personalidad y la educación en derechos humanos. Madrid: Pirámide.

Garaigordobil, M. y Garcia de Galdeano, P. (2006). Empatía en niños de 10 a 12 años. Psicothema, 18 (2), 180-186.

Gómez-Fraguela, J. A., Sobral, J., Luengo, M. A., Romero, E., y Villar, P. (2009). El mito del inmigrante delincuente. Boletín Criminológico, 112.

Hair, J. F., Anderson, R. E., Tatham, R. L., \& Black, W. C. (1999). Análisis multivariante. Madrid: Prentice Hall.

Hernández-Plaza, S., García-Ramirez, M., Camacho, C., \& Paloma, V. (2010). New settlement and well-being in oppresive contexts : A liberation psychology approach. En C. Stuart (Ed.), The psychology of global mobility. International and Cultural Psychology Series (pp. 235-256). New York: Springer.

INJUVE (2008). Informe de la juventud en España. Recuperado el 06 de febrero de 2011 de http://www.injuve.migualdad.es/injuve/portal. portal.action.

Jolliffe, D. \& Farrington, D. P. (2006). Development and Validation of the Basic Empathy Scale. Journal of Adolescence, 29, 589-611.

Luengo, M. A., Otero, J. M., Romero, E., Gómez-Fraguela, J. A., y Tavares, T. E. (1999). Análisis de ítems para la evaluación de la conducta antisocial: un análisis transcultural. Revista Iberoamericana de Diagnóstico y Evaluación, 1, 21-36.

Luengo, M. A., Villar, P., Sobral, J., Romero, E. y Gómez-Fraguela, J. A. (2009). El consumo de drogas en adolescentes inmigrantes: implicaciones para la prevención. Revista Española de Drogodependencias, 34, 4, 448479 
Luengo. A.. Carrillo. M. T.. Otero. J. M., \& Romero. E. (1994). A short-term longitudinal study of impulsivity and antisocial behavior. Journal of Personality and Social Psychology, 66, 542-548.

Marsiglia, F. F., Kulis, S., Wagstaff, D. A., Elek, E., \& Dran, D. (2005). Acculturation status and substance use prevention with mexican and mexican-american youth. Journal of Social Work Practice in the Addictions, 5(1-2), 85-111.

Olson, D. H., Portner, J. , \& Bell, R. Q. (1982). FACES II. St. Paul, MN: University of Minnesota.

ONU (2002). World immigration report. Nueva York: United Nations.

Prinz, R. J., Foster, S., Kent, R. N., \& O’Leary, D. (1979). Multivariate assessment of conflict in distressed and non distressed mother-adolescent dyads. Journal of Applied Behavior Analysis, 12 (4), 691-700.

Ramirez, M. D. C., Torrente, G., Ruiz, J. A. y Rodriguez, A. (2007). Análisis cualitativo de las atribuciones causales endo/exogrupales sobre los problemas relacionados con la inmigración: la perspectiva de los inmigrantes hispanoamericanos. En J. Romay (Coord.) Perspectivas y Retrospectivas de la Psicología Social en los albores del Siglo XXI (pp.363-370). Madrid: Biblioteca Nueva.

Redfield, R., Linton, R., \& Herskovits, M. (1936). Memorandum on the study of acculturation. American Anthropologist, 38, 149-152.

Romero, E., Luengo, M. A. y Gómez-Fraguela, J. A. (2000). Factores psicosociales y delincuencia: un estudio de efectos recíprocos. Escritos de Psicología, 4, 78-91.

Romero, E., Luengo, M. A., \& Sobral, J. (2001). Personality and antisocial behaviour: study of temperamental dimensions. Personality and Individual Differences, 31, 329-348.

Romero, E., Luengo, M. A., Gomez-Fraguela, J. A. y Sobral, J. (2002) La estructura de los rasgos de personalidad en adolescentes: El Modelo de los Cinco Grandes y los Cinco Alternativos. Psicothema, 14(1), 134-143.

Rudmin, F. W. (2003). Critical history of the acculturation psychology of assimilation, separation, integration and marginalization. Review of General Psychology, 7(1), 3-37.

Rudmin, F. W. (2006). Debate in science: the case of acculturation. AnthroGlobe Journal. Recuperado el 15 de mayo de 2010 de http://www.anthroglobe.ca/docs/rudminf_acculturation_061204.pdf.

Ruiz, J. A., Torrente, G. y Rodríguez, A. (2009). Actitudes de aculturación hacia inmigrantes hispanoamericanos y estrés de aculturación en autóctonos. XI Congreso Nacional de Psicología Social, Tarragona.

Silva, F., Martorell, M. C. , \& Clemente, A. (1987). I6 (junior) questionnaire: Spanish version. Psychological Assessment: An International Journal, 3, 55-78.

Sobral, J., Gómez-Fraguela, J. A., Luengo, A., Romero, E. y Villar, P. (2010). Adolescentes latinoamericanos, aculturación y conducta antisocial. Psicothema, 22(3), 410-415.
Sobral, J., Gómez-Fraguela, J. A., Romero, E., \& Luengo, M. A. (2000). Impulsividad, género y contextos, su interacción en la conducta antisocial. Anuario de Psicología Jurídica, 10, 79-91.

Sobral, J., Gómez-Fraguela, J. A., Luengo, M. A. y Romero, E. (2010). Inmigración: humanidades periféricas y humillación. A la búsqueda del "paradigma utópico mínimo". Psicología Política, 41, 27-41.

Sobral, J., Luengo, M. A., Gómez-Fraguela, J. A., Romero, E. y Villar, P. (2007). Personalidad, género y criminalidad violenta en reclusos. Psicothema, 19, 2, 269-275.

Sobral, J., Romero, E., Luengo, A. y Marzoa, J. (2000). Personalidad y conducta antisocial: amplificadores individuales de los efectos contextuales. Psicothema, 12 (4), 661-670.

Szapocznik, J., Kurtines, W., \& Fernández, T. (1980). Biculturalism and adjustment among Hispanic youths. International Journal of Intercultural Relations, 4, 353-375.

Torrente, G. \& Vazsonyi, A. T. (2008). The salience of the "family" in antisocial and delinquent behaviours among spanish adolescents. Journal of Genetic Psychology, 169, 187-197.

Vazsonyi, A. T. \& Belliston, L. M. (2007). The family, low self-control and desviance. A cross-cultural and cross-national test of Self-Control Theory. Criminal Justice and Behaviour, 34 (4), 505-530.

Vazsonyi, A. T., Trejos-Castillo, E., \& Huang, L. (2006). Are developmental processes affected by inmigration?. Family processes, internalizing behaviors, and externalizing behaviors. Journal of Youth Adolescence, 35, 799 813.

Villar, P., Luengo, M. A., Gómez-Fraguela, J. A., \& Romero, E. (2006). Assessment of the validity of parenting constructs using the multitraitmultimethod model. European Journal of Psychological Assessment, 22(1), 5968.

Villar, P., Luengo, M. A., Gómez-Fraguela, J. A., y Romero, E. (2003). Una propuesta de evaluación de variables familiares en la prevención de la conducta problema en la adolescencia. Psicothema, 15(4), 581-588.

Zarza, M. J., y Sobrino, M. I. (2007). Estrés de adaptación sociocultural en inmigrantes latinoamericanos residentes en Estados Unidos vs España: una revisión bibliográfica. Anales de Psicología, 23(1), 72-84.

Zuckerman, M., Kuhlman, D. M., Joireman, J., Teta, P., \& Kraft, M. (1993). A comparison of three structural models for personality: the big three, the big five, and the alternative five. Journal of Personality and Social Psychology, 65(4), 757-768.

(Artículo recibido: 13/4/2011; revisado: 19/10/2011; aceptado: 26/10/2011) 